\title{
A DESIGN FOR IMPROVING THE CORE SHELF SYSTEM IN A CORE REPOSITORY
}

\author{
X. Xing ${ }^{1}$, W.S.Hu ${ }^{1}$, L.Gao ${ }^{2}$ \\ ${ }^{1}$ School of Earth Sciences, Yangtze University, No. 1 Daxue Road, Wuhan, Hubei, China \\ ${ }^{2}$ Exploration and Development Research Institute of Huabei oilfield, Renqiu, Hebei, China \\ Corresponding Author Tel.: +86-027-69111922.; fax: +86-027-69111650. \\ justgoo1985@gmail.com
}

\begin{abstract}
Current core repository storage strategies and existing problems with current core rack retrieval were analyzed. Current retrieval methods were found to be inefficient both in the storage and retrieval of core samples. This prompted a study on improvements in storage algorithms. This new algorithm allows for improvements in rack structure, identification systems and databases used for core storage. This is done by utilizing structured information gathering for core storage areas, core observation areas and transportation devices and therefore can effectively improve the work efficiency in reducing work intensity and convenient data processing.
\end{abstract}

Keywords: Core repository, improvement, shelf system

\section{INRODUCTION}

Cores are rock samples obtained by drilling into ores. Core whole locations are determined by using stratigraphic horizon and depth of stratum techniques. The use of cores is helpful in both the process of studying and developing oil fields and in the location of solid mineral deposits. They are also useful in determining the physical and chemical composition of underground deposits. In addition, they are important geological archives which need specialized facilities for longterm preservation and study.

In view of the importance of core sampling, China's relevant departments were tasked with performing pertinent experiments as well as with the study of foreign reports. Core repositories specialize in storing core samples. Using existing cold storage techniques, a core is stored in a single unit in a core rack. Due to the number of core samples for any given well, a core rack may contain numerous samples from different sources. Records are maintained for the location of each core section, additionally tagged with each core sample's source well. When a core rack contains many samples from several wells, the sample must be removed in a timely manner when testing of the sample has been scheduled and then transported to an observation room. Each core box contains multiple samples, a single row of which, when fully loaded, can weigh in excess of $15 \mathrm{~kg}$, with a double row of core boxes weighing in excess of $30 \mathrm{~kg}$. Loading and unloading of core samples by hand can become onerous due to the weight of core boxes, so carrying robots are typically used to retrieve core samples. Despite the benefits provided by automatic retrieval, there are several disadvantages to using this method.
Disadvantages include the complex operation and difficult maintenance of the equipment, a lack of refined categorization techniques, high maintenance costs and equipment fragility. Each adds to the cost of core sample retrieval and testing processes.

Using existing core testing procedures, manual preparation and core location account for $40 \%$ of total work time, with the remaining $60 \%$ used for the testing itself. This increases the intensity of the work while lowering its efficiency. With an increasing workload, more time for manual preparation and core location will be required. By achieving an improvement in the core retrieval process, more time can be allotted for testing samples and observation.

\subsection{DESIGN PROCEDURE}

\subsection{Analyzing the Storage and Removal Work for}

\section{Core Boxes from a Core Rack}

Storage and removal of core boxes involve separate procedures, with similar methods for achieving the intended results. The first step in any storage or retrieval is performed by querying the location for the box. The robot will then locate the box given this information and then retrieve the box. The time spent on storage or retrieval can be divided into three parts, representing each of these steps. Formula 1 can then be given as follows:

$$
\mathrm{T}_{\mathrm{qc}}=\mathrm{T}_{\mathrm{cx}}+\mathrm{T}_{\mathrm{dw}}+\mathrm{T}_{\mathrm{qz}}
$$


where Tqc is the total time, Tcx represents the time spent on the database query, Tdw is the location time and Tqz is the time spent on retrieval (or storage). Measures that can be used to improve the efficiency in each of these three time periods will improve overall efficiency as well.

In order to obtain the location of a specific core box, the major position and specific location of it must be known. The time spent on a given inquiry can be further subdivided into querying the two individual parts of this data set.

$$
\mathrm{T}_{\mathrm{cx}}=\mathrm{T}_{\mathrm{qw}}+\mathrm{T}_{\mathrm{dd}}
$$

Where Tqw represents the major position query time and $T_{d d}$ is the specific location query time.

Measures improving the efficiency of the query in either mode can be then said to improve overall efficiency.

\subsection{Analyzing Workload For Removal Of Core Boxes}

To determine the removal work for a targeted core box, we should first look at the position of the targeted box on a rack. If each frame core box can be said to have n layers, and each layer has $\mathrm{m}$ core boxes, and both $\mathrm{n}$ and $\mathrm{m}$ are elements of $\mathrm{N}+$, as in Formula 3,

$$
\mathrm{N}+
$$

and the position $(\mathrm{P})$ is a function of $\mathrm{m}$ ' and $\mathrm{n}$ where

$$
\mathrm{m}^{\prime}=1,2,3,4, \ldots 1 / 2 \mathrm{~m}
$$

Under normal operation, the manipulator always moves from a larger to a smaller number. If $\mathrm{m}$ ' $>1 / 2 \mathrm{~m}$, the manipulator will take core samples from the opposite side of a core box. By design, $m$ ' is always counted from the end of the rack with the fewest number of core boxes.

Since core boxes are removed from top to bottom with the uppermost boxes being removed first, $\mathrm{n}$ is counted from this direction as well. When the target box is at the top layer of the rack $(n=1)$, the manipulator removes that number according to the following algorithm:

$$
\begin{aligned}
& \mathrm{Y}=\mathrm{m}^{\prime} \text { for } m^{\prime} \leq 1 / 2 m \\
& \text { If } \mathrm{n}>1 \text { and } \mathrm{m}>1, \text { then } \mathrm{Y}=\mathrm{n}^{*} \mathrm{~m}+1 / 2(\mathrm{n}-1)\left(\mathrm{m}-\mathrm{m}^{\prime}\right) \\
& \quad \text { for } P=1,2,3, \ldots 1 / 2 \mathrm{~m}
\end{aligned}
$$

For example, if $n=1$ and $m=10$, then $m^{\prime}=5$, determining the number of boxes the manipulator should remove are simply:

$$
\begin{aligned}
& \mathrm{Y}=\mathrm{m}^{\prime}\left(\text { because } \mathrm{m}^{\prime} \leq 1 / 2 \mathrm{~m}\right) \\
& \mathrm{Y}=5
\end{aligned}
$$

So, if $\mathrm{n}=5, \mathrm{~m}=10$ and $\mathrm{P}=5$, the number of core boxes the manipulator should remove will be:

$$
\begin{array}{ll} 
& \mathrm{n}>1, \mathrm{~m}>1, \mathrm{P} 1 / 2 \mathrm{~m} \\
& \mathrm{Y}=\mathrm{n}^{*} \mathrm{~m}^{\prime}+1 / 2(\mathrm{n}-1)\left(\mathrm{m}-\mathrm{m}^{\prime}\right) \\
\text { or } & \mathrm{Y}=5 * 5+1 / 2(5-1)(10-5)=35 .
\end{array}
$$

As layers in the core rack are removed, the number of boxes the manipulator will be required to remove to reach a specific location will be reduced. When the number of the core box reaches 1 , the quantity the manipulator will need to remove is defined as $Y^{\prime}$, where $Y^{\prime}=Y(n=1)$.

$$
\begin{aligned}
& Y^{\prime}=1 * m+1 / 2(1-1)(10-5)=5 \\
& Y^{\prime} Y^{\prime}=30
\end{aligned}
$$

This gives a $14 \%$ reduction in the workload when $n=1$, which demonstrates that increasing the number of layers is more effective than increasing the number of core boxes in a single layer.

\subsection{Reducing The Friction Of Core Boxes During}

\section{Storage And Removal}

Since rolling has a lower coefficient of friction than sliding, the use of sliding friction by installing a tray and pulley system can be used to increase efficiency in the removal process. This allows for the separation of different core boxes in distinct positions in the core rack. Using four rollers corresponding to the added trays will help realize this substitution. To accommodate the extra weight of the trays, additional thickness in the baseboards of the rack should be adopted, as well.

\subsection{Improving the Recognition of Core Boxes}

Using formulas 1 and 2, a corresponding logo for each box with an accompanying location device should be used to decrease the time needed to locate targeted boxes.

\section{IMPROVED DESIGN PROPOSAL}

\subsection{Improvement In Rack Layering of Core Boxes}

According to the example given in section 2.2, reducing the number of the layers in core racks improves the efficiency of storage and removal work. In order to achieve this improvement, the structure of the core shelves must be altered by reducing the distance between each rack used for core storage. Using a shelf which houses a monolayer instead of shelving which contains multiple layers and multiple boxes can achieve the desired improvements. Additional improvements can be realized by placing the same core 
samples from identical core holes adjacent to one another as shown in Fig. 1 below.

\subsection{Reducing The Friction Of Core Boxes During}

\section{Storage And Removal}

To reduce the resistance of storing and removing core boxes, each pallet is designed on the basis of a single core box space, with each core box tray installed on the chassis of a wheel having inlets and outlets fixed on both sides of the storage racks. This allows for easier removal of core boxes due to decreased friction at the box/tray interface as shown in Picture 2 below.

\subsection{Improving Core Box Identification Efficiency}

To improve the efficiency of identifying core boxes, a core rack identification system needs to be established. Using a laser digital ID system to tag both ends of the box (Figure 3), each box can be labeled with its own digital ID. Attaching the computer database to each shelf with the digital information for cores contained in a given shelf increases the efficiency of storage and removal by allowing for a unified management of the repository as shown in Picture 3 below.

Integrating all core store data in a single database for the core storage area, core observation area, scanning area and access forklift for each storage rack forms an organic whole. The forklift computer and the control room computer are linked by an uninterrupted signal offering complete access of core data to the forklift computers.

In order to implement these improvements, core numbers need to be entered into the control room computer first. The forklift computers access this data thereby retrieving the correct core box from the storage shelving area and bringing it to the transportation tracks which lead to the core library. The targeted cores are then moved to the core observation area.

\section{SUMMARY OF IMPROVEMENTS}

\subsection{Effects Of Improvements}

Improved access and efficiency for core boxes: Compared with the original design for the core shelf, the improved design can reduce the workload and improve core box access rates. Added efficiency is observed the further a box is located down in the core rack. For a target core box at a depth of five layers under the original configuration, the access rate using the improved method requires $14 \%$ of the time needed without any improvements. With a reduction in the effects of friction during the handling process also incorporated, the time to retrieve a target core box will be reduced even further.

Improved query efficiency for core boxes: With improved tagging on core boxes and shelves entered into a database located in the observation room, the computer can be used to make a quick inquiry about the target box location by using localized shelf location information kept at each core rack. This significantly improves the efficiency of location queries. Security Issues: Incorporating a tray design into the improved core rack system allows for the placement of core boxes directly into each tray. This eliminates the problem of collapsing racks due to the overtop centre of gravity caused by a single tray with multi-layer core boxes and uneven weight distributions.

\subsection{Experiences with Improvements}

Innovations introduced by this product: Recognizing the deficiencies of existing products is an important way to improve the products. By observing existing production lines carefully, a problem can be isolated.

Improvements of product performance: The disruptive effect of continuing to use existing methods in production is relevant when justifying the adoption of new techniques and technologies. After determining the cause of a product's deficiencies and addressing these, overall efficiency of a process can be improved as well.

\section{REFERENCES}

[1] Xiang-dong Liu, Li-hai Zhang, et al. On China's Physical Geological Data Management and Social Services [J]. Land and Resource Technology Management 2010, 27 (2): 133-136.

[2] Xin Yan. China National Committee for Terms in Sciences and technologies [DB/OL]. http://baike.baidu.com/view/1746161.htm

[3] Xin-yu Chen, Qing-min Nan. The Management and Services of Russian Physical Geological Data [J]. Natural Resource Economics, 2011 (8): 25-28, 55.

[4] Yan Li, the Brief Exposition of Classification and Arrangement of the Fifteen Geological Data [J]. Geology, 2009, 27 (2): 198-199.

[5] Yan-ping Liu, The Management of Russian Geological Data [J]. Land and Resources Information, 2006 (11): 8-13. 


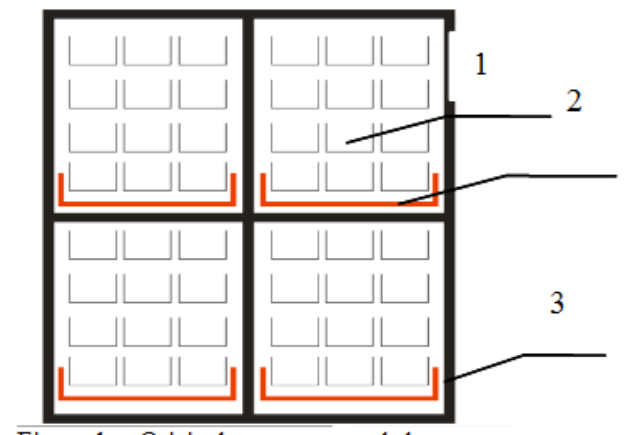

Figure 1 a. Original core storage shelves.

In Figure 1 a: 1 ---The core box of the original core storage shelves

2 ---- The core box pallets of the original core storage shelves

3 ----The original core storage shelves

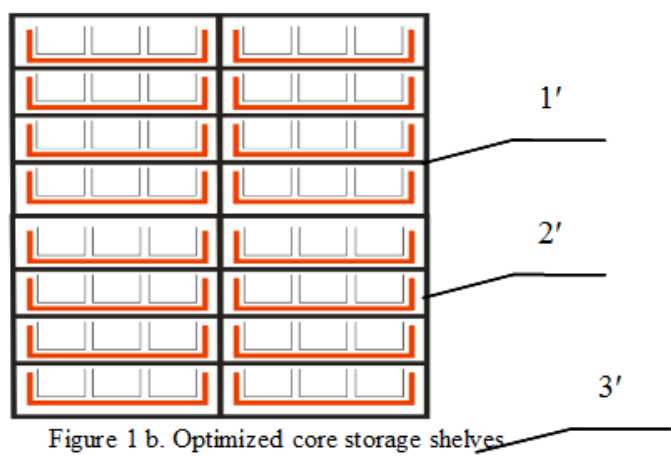

b: $1^{\prime}-$ The core shelves $2^{\prime}$---The core box pallets of the optimized core storage shelves $3^{3}$---The optimized core storage shelves

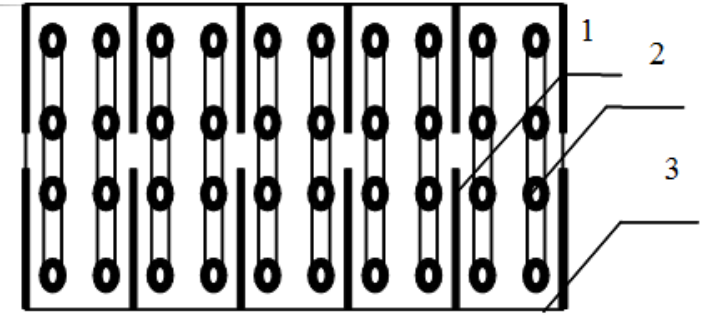

Picture 2a. Pictorial diagram of core shelf

In picture $2 \mathrm{a}$ : 1--every clapboard of core box

2--pulley on shoot of core box 3--shoot of board on core box

Picture 2 modified stored board shelf of core boxes, structure of pulley and enhancement structure of core box floor.

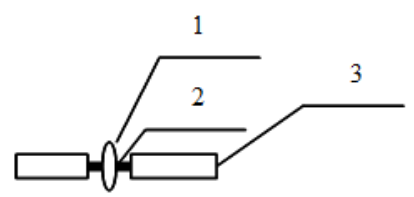

Picture $2 \mathrm{~b}$. Plane pulley schedule of shelf in core In picture $2 \mathrm{~b}: 1$--pulley on board store at core box

2 - poppet head on stored board install at core

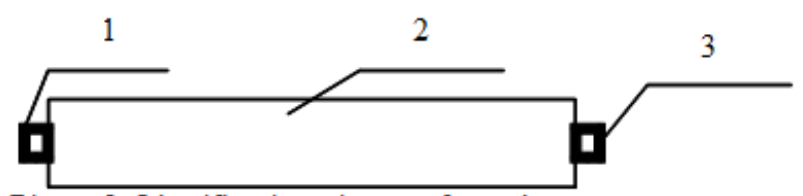

Picture3. Identification picture of core box

In picture3: 1, 3--laser mark get out from core box

2--core box

3--laser identification deposit in core box 